\title{
ON THE FUNDAMENTAL GROUPS OF MANIFOLDS WITH ALMOST-NONNEGATIVE RICCI CURVATURE
}

\author{
GUOFANG WEI
}

(Communicated by Jonathan M. Rosenberg)

\begin{abstract}
We give an upper bound on the growth of $\pi_{1}(M)$ for a class of manifolds $M$ with Ricci curvature $\operatorname{Ric}_{M} \geq-\varepsilon$, diameter $d(M)=1$, and volume $\operatorname{vol}(M) \geq v$.
\end{abstract}

In [4], Milnor proved that every finitely generated subgroup of the fundamental group of a manifold $M^{n}$ with nonnegative Ricci curvature is of polynomial growth with degree $\leq n$. It is conjectured by Gromov [2] that the fundamental group of a near-elliptic manifold (in the sense of Gromov) is of polynomial growth. The purpose of this note is to present the following theorem.

Theorem 1. For any constant $v>0$, there exists $\varepsilon=\varepsilon(n, v)>0$ such that if a complete manifold $M^{n}$ admits a metric satisfying the conditions $\operatorname{Ric}_{M} \geq-\varepsilon$, $d(M)=1$, and $\operatorname{vol}(M) \geq v$, then the fundamental group of $M$ is of polynomial growth with degree $\leq n$.

Our proof depends essentially on a recent result of M. Anderson [1].

Theorem 2 (M. Anderson). In the class of compact $n$-dimensional Riemannian manifolds $M$ such that $\operatorname{Ric}_{M} \geq(n-1) H, \operatorname{vol}(M) \geq v$, and $d(M) \leq D$, there are only finitely many isomorphism classes of $\pi_{1}(M)$.

Proof of Theorem 1 . Choose a base point $\tilde{x}_{0}$ in the universial covering $\widetilde{M} \stackrel{p}{\rightarrow}$ $M$, and let $x_{0}=p\left(\tilde{x}_{0}\right)$ and $g_{1}, \ldots, g_{r}$ be a set of generators of the fundamental group $\pi_{1}(M)$ viewed as deck transformations in the isometry group of $\widetilde{M}$. Denote $\Gamma(s)=\left\{\right.$ distinct words in $\pi_{1}(M)$ of length $\left.\leq s\right\}, \gamma(s)=\# \Gamma(s)$, and $l=\max _{1 \leq i \leq r}\left\{d\left(\tilde{x}_{0}, g_{i}\left(\tilde{x}_{0}\right)\right)\right\}$.

Choose a fundamental domain $F$ of $\pi_{1}(M)$ which contains $\tilde{x}_{0}$; then

$$
\bigcup_{g \in \Gamma(s)} g(F) \subset B_{s l+d}\left(\tilde{x}_{0}\right),
$$

where $d=d(M)=1$. Therefore,

$$
\gamma(s) \cdot \operatorname{vol}(M) \leq \operatorname{vol}\left(B_{s l+1}\left(\tilde{x}_{0}\right)\right)
$$

Received by the editors March 13, 1989 and, in revised form, October 23, 1989.

1980 Mathematics Subject Classification (1985 Revision). Primary 53C20; Secondary 57S20.

This research was supported in part by Alfred P. Sloan Doctoral Dissertation Fellowship. 
Now suppose, on the contrary, that for any $\varepsilon>0$, there is a manifold $M^{n}$ with a metric satisfying $\operatorname{Ric}_{M} \geq-\varepsilon, d(M)=1, \operatorname{vol}(M) \geq v$, and $\pi_{1}(M)$ is not of polynomial growth with degree $\leq n$. By the proof of Theorem $2, \pi_{1}(M)$ has a presentation which obeys the following:

(1) The number of generators $g_{1}, \ldots, g_{N}$ is uniformly bounded with $N \leq$ $N\left(v / D^{n}, H D^{2}\right)$,

(2) $d\left(g_{i}\left(\tilde{x}_{0}\right), \tilde{x}_{0}\right) \leq 3 D$,

(3) every relation is of the form $g_{i} g_{j}=g_{k}$.

The statements (2) and (3) have already been proved by Gromov [3]. By our assumption, $\pi_{1}(M)$ is not of polynomial growth with degree $\leq n$. In particular, when taking the above generators, we can find real numbers $s_{i}$ for all $i$ such that

$$
\gamma\left(s_{i}\right)>i s_{i}^{n}
$$

It is crucial that this relation is independent of $\varepsilon$, as follows from (1) and (3).

On the other hand, by (1) we have

$$
\gamma(s) \leq \frac{1}{v} \int_{0}^{3 s+1}\left(\frac{\sinh \sqrt{\varepsilon t}}{\sqrt{\varepsilon}}\right)^{n-1} d t .
$$

For any fixed, sufficiently large $s_{0}$, there is $\varepsilon_{0}=\varepsilon\left(s_{0}\right)$ such that for all $s \leq s_{0}$, $\varepsilon \leq \varepsilon_{0}$,

$$
\gamma(s) \leq \frac{6^{n}}{n v} s^{n} .
$$

Now take $i_{0}>6^{n} / n v$. Then for $\varepsilon<\varepsilon\left(s_{i_{0}}\right)$, using (2) and (3), we get a contradiction.

We would like to mention that Peter Peterson, working from a different orientation and with different technique, has obtained a slightly weaker result. Instead of a lower volume bound, he imposes a lower bound on the contractibility radius and arrives at the same conclusion.

\section{ACKNOWLEDGMENTS}

The author would like to thank Detlef Gromoll and Michael Anderson for very helpful conversations.

\section{REFERENCES}

1. M. Anderson, Short geodesics and gravitational instantons, J. Differential Geom. 31 (1990) (to appear).

2. M. Gromov, Synthetic geometry in Riemannian manifolds, Proc. ICM 1978, Helsinki (1980), 415-421. 
3. __ Structures métriques pour les variétés Riemanniennes, Cedic Fernand-Nathan, Paris, 1981.

4. J. Milnor, A note on curvature and fundamental group, J. Differential Geom. 2 (1968), 1-7.

Department of Mathematics, State University of New York at Stony Brook, Stony BROOK, NEW YORK 11794-3651

Current address: Department of Mathematics, Massachusetts Institute of Technology, Cambridge, Massachusetts 02139 